\section{Boosting cancer immunotherapy}

CD47 is an antiphagocytic 'don't eat me' signal highly expressed by many cancer cells to avoid detection by macrophages. In Science, Weiskopf et al. use a high-affinity, in vitro-engineered variant of SIRPa, the ligand for CD47, to antagonize CD47 on cancer cells. The SIRPa variant competes with wild-type SIRPa for the same epitope, and binds human CD47 with greater affinity and has stronger antagonism than does wild-type SIRPa. The high-affinity SIRPa monomers do not induce macrophage phagocytosis on their own but act in synergy with tumor-specific antibodies to induce phagocytosis of tumor cells in vitro. In vivo, the high-affinity SIRPa monomers act as an adjuvant for tumor-specific antibodies to enhance macrophage infiltration, inhibit tumor growth and improve survival in various tumor models and have no cytotoxicity against untransformed cells that express CD47. Thus, these engineered SIRPa variants may provide a universal adjuvant for augmenting the efficacy of cancer immunotherapies.

Science (30 May 2013) doi:10.1126/science.1238856

\section{Integration-induced death}

The mechanism by which human immunodeficiency virus type 1 (HIV-1) induces the death of helper $\mathrm{T}$ cells has not been defined. In Nature, Cooper et al. show that virus-induced cell killing is triggered by viral integration and involves activation of the DNA damage-response protein DNA-PK. Through the use of an integrase-deficient HIV-1 mutant virus and integrase inhibitors, the authors show that integration is both necessary and sufficient to trigger cell death by HIV-1. Integration elicits a cellular response to double-stranded DNA damage involving activation of DNA-PK, phosphorylation of the tumor suppressor p53 and cell death. A pharmacological integrase inhibitor abolishes HIV-1-induced killing of primary $\mathrm{CD}^{+} \mathrm{T}$ cells from acutely infected subjects, and inhibition of DNA-PK abolishes cell death during HIV-1 infection in vitro. These results suggest that inhibitors of these enzymes may improve T cell survival and immunological function in HIV-1-infected people.

IV

Nature (5 June 2013) doi:10.1038/nature12274

\section{NO to Leishmania}

Leishmaniasis, a prevalent parasitic disease that causes considerable morbidity, is dampened by the production of nitric oxide (NO) by macrophages. In Nature Medicine, Zamboni and colleagues show that the NLRP3 inflammasome is activated after infection with Leishmania and is required for optimal production of NO. Live parasites trigger assembly of NLRP3 inflammasomes, caspase- 1 activation and release of IL-1 $\beta$, but dead parasites do not. Signaling through the IL-1 $\beta$ receptor via MyD88 acts in synergy with IFN- $\gamma$ to enhance expression of the gene encoding inducible NO synthase and elicit NO production. Mice deficient in the inflammasome components NLRP3, Asc or caspase-1 have a greater parasite burden, similar to that of mice that lack NO synthase. It remains unclear how Leishmania parasites trigger inflammasome activation; however, it probably involves active lysosomal membrane damage triggered by the parasite.

\section{Barrier with a bite}

The intestinal epithelium has a key role in limiting the dissemination of gut microbes through both its function as a physical barrier as well as its release of antimicrobial peptides. In Cell Host and Microbe, Hooper and colleagues demonstrate that bacteria-induced autophagy in intestinal cells is important for gut immunity. Challenging mice with invasive Salmonella serovar Typhimurium or Entercoccus faecalis triggers autophagy in the intestinal epithelium. The ability to invade the epithelium is critical for such a response, because the normal host microbiota or mutant bacteria unable to enter cells fail to elicit autophagy. Signaling via the pattern-recognition receptor adaptor molecule MyD88 in epithelium is also essential, but how this is 'wired' into the autophagocytic machinery is unclear. Finally, defective autophagocytosis specifically in intestinal epithelium leads to more dissemination of bacteria. Thus, epithelial cell-intrinsic autophagy is a further means by which gut barrier integrity is maintained.

Cell Host Microbe (12 June 2013) doi:10.1016/j.chom.2013.05.004

\section{Metabolic control of cytokines}

Full effector function of $T_{H} 1$ cells requires that the cells switch to aerobic glycolysis. In Cell, Pearce and colleagues report post-transcriptional regulation of mRNA encoding IFN- $\gamma$ and IL-2 mediated by the enzyme GAPDH when cells switch their metabolism from oxidative phosphorylation to aerobic glycolysis. GAPDH binds to AU-rich elements in the $3^{\prime}$ untranslated region of $\mathrm{mRNA}$ to suppress protein translation. That effect is relieved by increasing the abundance of glyceraldehyde-3phosphate, the GAPDH substrate produced during glycolysis. Studies of T cells with transgenic expression of green fluorescent protein-linked GAPDH, isolated from mice infected with Listeria monocytogenes, show that the expression of IFN- $\gamma$ and IL-2 protein is inversely correlated with GAPDH abundance. Interestingly, expression of the inhibitory molecule PD-1 is higher in T cells with higher expression of GAPDH. The authors suggest that the availability of glucose as a carbon source serves to regulate $T$ cell effector function by influencing GAPDH activity as either a post-transcriptional regulator or a catalytic enzyme in the glycolysis pathway.

\title{
Evolving away
}

Viruses use specific host receptors to infect cells, and this frequently triggers an evolutionary 'arms race', with the host mutating receptors and the virus playing 'catch-up. In PLOS Biology, Sawyer et al. investigate how the host counters viruses that use highly conserved host receptors for entry. The transferrin receptor Tfr1 is used by certain viruses for entry into host cells, but because Tfr 1 is critical for cell survival, it does not tolerate mutation well. By examining the structures and sequences of Tfr1 from different rodent species, the authors find that the entire receptor is highly conserved except for a few residues that undergo strong positive selection. Those sequences overlap the interaction surfaces of arenaviruses and mouse mammary tumor viruses that use Tfr 1 for entry and thereby impose strict barriers on infection. These findings offer insight into how the host can mutate critical housekeeping genes to impede the entry of viruses without affecting essential physiological functions. PLOS Biol. (28 May 2013) doi:10.1371/journal.pbio.1001571 